\title{
TCAD Device Simulations of Irradiated Silicon Detectors
}

\section{F.R.Palomo*}

School of Engineering, U. of Sevilla, Spain

E-mail: fpalomo@us.es

\section{M.Moll}

CERN, Geneva, Switzerland

E-mail: michael.mollecern.ch

\section{J.Schwandt}

Institute of Experimental Physics, U.of Hamburg, Germany

E-mail: joern.schwandt@desy.de

\section{E.Giulio Villani}

STFC Rutherford Appleton Laboratory, United Kingdom

E-mail: Enrico.Giulio.Villani@cern.ch

\section{Y.Gurimskaya}

CERN,Geneva, Switzerland

E-mail: yana.gurimskaya@cern.ch

\section{R.Millán}

School of Engineering, U. of Sevilla, Spain

E-mail: rmillan@us.es

The high hadron fluences expected during the HL-LHC programme will impose strong constraints in terms of radiation damage on silicon detectors. New TCAD simulation models are needed to predict the expected detector performances. This review examines the challenges ahead for different types of detector devices, with emphasis on the acceptor removal effect in LGADs, surface damage in Monolithic and Strip sensors and bulk damage in all sensor types.

The 28th International Workshop on Vertex Detectors - Vertex2019

13-18 October, 2019

Lopud, Croatia

\footnotetext{
${ }^{*}$ Speaker.
} 


\section{Introduction}

To observe rare high energy physics (HEP) processes we need large statistical datasets which means a high particle-flux environment. The detectors have to withstand the cumulated radiation damage, maintaining their quality of operation for years. The High Luminosity LHC (HL-LHC) programme, an upgrade of the existing LHC expected to start operation in 2027, will have an integrated luminosity of $4000 \mathrm{fb}^{-1}$ over 10 years of operation ${ }^{1}$. For the innermost pixel sensors, for a typical radii of $3 \mathrm{~cm}$, over their lifetime, this would translate into an expected hadron fluence ${ }^{2}$ around $2 \times 10^{16} \mathrm{n}_{\mathrm{eq}} / \mathrm{cm}^{2}$ and ionizing doses about $1 \mathrm{Grad}$ [1]. The RD50 collaboration is focused on the development of new solid state particle detectors for HL-LHC upgrade and possibly beyond (FCC programme).

One of the tools to predict the performances of detectors and to improve their design in such a harsh radiation environment is Technology Computer-Aided Design (TCAD) device simulation. A silicon particle detector can be viewed as the solid state analogue of an ionization chamber: the electric field strength is critical to separate the electron-hole pairs created by ionization, avoiding their recombination. The differentiation with ionization chambers comes in the carrier transport, governed by the laws of the crystal drift-diffusion model (the simplest one, but thermal and hydrodynamic carrier transport could also be considered). Using finite element analysis in a geometrical device model, the simulation solves the combined partial differential equations of charge continuity for charge transport and the Poisson equation for the electrostatic potential. In general, radiation damage modifies silicon detector performance; TCAD aims to predict such changes by means of I-V/C-V (current vs voltage, capacitance vs voltage), charge collection efficiency (CCE) simulations and predicts the signal shape, which can be used as input to the front-end ${ }^{3}$. Radiation damage affects the carrier generation-recombination mechanism, the charge carriers mobility and also the electric field by means of charge accumulation in dielectrics and traps.

In order to make reliable HEP device simulations, a thorough understanding of crystal defects introduced by Displacement Damage Dose (DDD) and Total Ionization Dose (TID) is necessary. TID effects appear when the incident particles lose energy by ionization (Ionizing Energy Loss, IEL) and can be described as purely electrostatic due to accumulation of holes in device dielectrics and the appearance of defects in the semiconductor-dielectric interface. Usually, TID effects are the most relevant in MOS electronics while displacement damage is more relevant in solid state detectors. Device simulation can easily deal with electrostatic effects, simply by considering new charge densities for the Poisson equation.

Displacement damage appears when the incident particles lose energy by non-ionizing mechanisms (Non Ionizing Energy Loss, NIEL) and is a complex problem because it affects not only the Poisson equation (by adding new charge densities due to the carrier trapping by defects) but also the carrier transport equations, specifically carrier mobility and the generation-recombination terms. The LHC radiation environment consists of $\gamma$ rays, electrons, and hadrons (mainly pions,

\footnotetext{
${ }^{1}$ One inverse femtobarn $\left(\mathrm{fb}^{-1}\right)$ corresponds approximately to $8 \times 10^{13}$ proton-proton interactions, assuming a proton inelastic cross section of $80 \mathrm{mb}$. The instantaneous luminosity of the HL-LHC is $5 \times 10^{34} \mathrm{~cm}^{-2} \mathrm{~s}^{-1}$ or at least five times the LHC instantaneous luminosity. The center of mass energy, $\sqrt{s}$ is $14 \mathrm{TeV}$.

${ }^{2}$ Integrated flux in terms of $1 \mathrm{MeV}$ equivalent neutrons for displacement damage

${ }^{3}$ in mixed mode simulations TCAD can also include the first stage of the front-end as a circuit simulation
} 
protons and neutrons). The amount and type of crystalline damage is different for every particle type [2]. Radiation produces a distribution of primary knock-on atoms (PKAs) displaced from their lattice positions. The minimum energy in silicon necessary to create a Frenkel pair (vacancy plus separated Si interstitial) or point defect displacement energy, is $\sim 25 \mathrm{eV}$. The threshold energy to produce densely packed displacement regions (clusters) is $\sim 5 \mathrm{keV}$. Gamma rays generate secondary Compton electrons responsible for a recoil PKA spectrum that peaks at $<1 \mathrm{keV}$ so point defects are dominant. For electrons, the PKA spectrum depends on the initial energy, for example, $15 \mathrm{MeV}$ electrons produce a maximum PKA energy of $250 \mathrm{keV}$ so defect clusters will also appear. Coulomb interaction is the main mechanism of energy loss by charged hadrons so the PKA spectrum extends from point defects (low energy) to defect clusters (large energy). Finally, neutron interactions are dominated by head-on collisions; for a $1 \mathrm{MeV}$ neutron the mean energy transfer to a $\mathrm{Si}$ atom is $\sim 50 \mathrm{keV}$ so they will produce a high density of clusters.

A further complication arises from the fact that only a subset of defects is important: those responsible for the macroscopic effects at the device functional level, as active electrical defects (also known as traps). The traps are able to modify the charge transport (see for example [3] for an updated list of traps in $\mathrm{Si}$ ): by reducing the carrier mobility or by deactivating dopants via kickout reactions (particularly relevant for the acceptor removal effect). Traps produce charge trapping and changes in the leakage current, $I_{\text {leak }}$ and in the effective space charge density, $N_{\text {eff }}$, see Fig.1. Higher leakage current is esentially produced by the defects with energy levels close to the middle of the bandgap. Increased leakage current implies more noise and more power consumption at the device level.

The effective space charge density $N_{\text {eff }}$ in undamaged detectors is just the bulk doping. In damaged detectors, $\Delta N_{\text {eff }}$ is mainly due to charged defects: acceptors in the lower half of the bandgap tend to contribute with negative space charge and donors in the upper half tend to contribute with positive space charge. The $\Delta N_{\text {eff }}$ will shift the depletion voltage value (because $\left.V_{d e p}=e\left|N_{e f f}\right| d^{2} / 2 \varepsilon \varepsilon_{0}\right)$ and will also change the electric field configuration within the device. A non-homogeneous space charge distribution can lead to new effects such as the occurrence of a double junction, possible underdepletion or shift of the electric field maximum to unwanted device regions. If the electric field locally exceeds $300 \mathrm{kV} / \mathrm{cm}$, impact ionization phenomena appear that could lead to a device breakdown.

Charge trapping by defect levels (donors and acceptors) reduces the available carriers. If the concentration of trapping centers and the detrapping time is long compared to the detector signal collection, the device will have a reduction in the Signal to Noise Ratio (SNR) and in the CCE. For defect clusters, a charge transfer appears between levels of neighbouring defects, also known as inter-centre charge (ICC) transfer [4]. This mechanism is beyond the Shockley-ReadHall (SRH) mechanism where carriers are captured by a single defect level. A first approach to inter-centre charge transfer is the coupled defect-level (CDL) recombination, available in TCAD [5] that considers transitions between two defect levels. Further modelization of ICC is beyond standard TCAD although there are efforts [6], to define new recombination models to be included in $\mathrm{TCAD}^{4}$. ICC is most visible in the increase of the leakage current so it can be taken into account,

${ }^{4}$ TCAD software packages include the possibility that advanced users can add new functions by means of dynamically linked libraries. 
in an ad-hoc manner, as an enhancement of the SRH generation rate [7] (the usual model for leakage current density is $J_{\text {leak }}=q G_{S R H}$ ).

For bulk p-type devices radiation damage shows two effects: a removal of the initial acceptor density and the appearance of usually acceptor-like defects due to the creation of the deep traps [8] shown in Eq. (1.1) :

$$
N_{e f f}(\Phi, x)=g_{e f f} \Phi+N_{A}(0, x) e^{-c \Phi}
$$

where $N_{A}(0, x)$ is the initial acceptor density, $\Phi$ is the radiation fluence $\left(1 \mathrm{MeVn}_{\text {eq }} / \mathrm{cm}^{2}\right), g_{\text {eff }} \sim$ $0.02 \mathrm{~cm}^{-1}$ is the introduction rate and $c$ is the acceptor removal constant (in a particular parameterization [8] c can be understood as $c=1 / \Phi_{0}$, where $\Phi_{0}$ is the fluence needed to reduce $N_{A}(0, x)$ by a factor of $1 / e$ ). The acceptor removal effect [9] is responsible for a deactivation of the initial boron dopant density because boron atoms are displaced from the substitutional lattice site and deactivated as shallow dopants. It is still under study [10] but the measurements are in agreement with the deactivation of boron atoms via the formation of ion-acceptor complexes, in a two step process: (i) the radiation induced creation of an interstitial $\mathrm{Si}$ atom and (ii) the deactivation of the boron via a Watkins kick-out reaction. The amount of removed boron does not change with long term annealing close to room temperature.

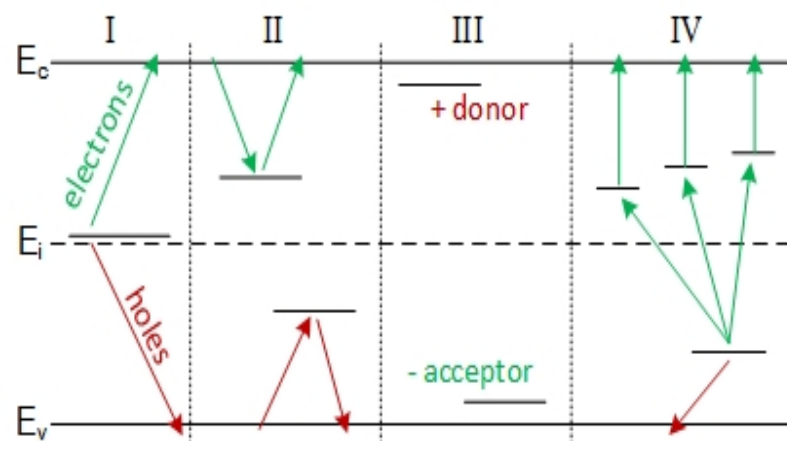

Figure 1: I. Increase of leakage current, II. Charge Trapping and CCE, III. Change of internal field and depletion voltage, IV. Enhanced generation by inter-center charge transfer. Defects energy levels are referenced to the Intrinsic Fermi level, $E_{i}$ and to the valence and conduction bands energies, $E_{V}, E_{C}$.

To summarise, radiation damage will degrade the device performance. Bulk damage by NIEL relates to point and cluster defects in the silicon lattice. As a consequence the device will show a change in $I_{\text {leak }}$ and $V_{\text {dep }}$, modifications in the electric field configuration and trapping of drifting charge. The trapping will reduce the CCE and reduce the SNR. Those effects are relevant for pad sensors and DC coupled strips and pixel detectors. For LGAD-type sensors [11] acceptor removal is responsible for gain reduction. Surface damage produced by NIEL is responsible for the build up of dielectric (oxide) charges and border and interface traps. It results in an increase of the surface current, the change of the electric field and charge trapping near the semiconductor ( $\mathrm{Si}$ )dielectric $\left(\mathrm{SiO}_{2}\right)$ interface. Surface damage is relevant in MOS capacitors, MOSFET transistors and gate controlled diodes, AC coupled detectors and particularly in MAPS/HVCMOS [12] detectors because they combine bulk detectors with MOSFET electronics in the same die.

Section 2 is dedicated to the TCAD modeling of radiation damage, Section 3 shows the most important high fluence TCAD defect models including the particularization of acceptor removal 
for LGAD type detectors and Section 4 summarizes different simulation examples with emphasis on MAPS detectors.

\section{TCAD modeling of radiation damage}

TCAD software tools are designed for physical simulation of the charge transport and electrical behavior of semiconductor devices. There are various commercial packages available, for example Silvaco Atlas or Synopsys Sentaurus. TCAD device simulation implements the semiclassical approach to electronic transport in semiconductors ${ }^{5}$. The most used set of equations [13] considers the Poisson equation (2.1) for the electric field, dependant on the instantaneous charge density, the current continuity in a semiconductor, Eqs.(2.2), (2.3), and the traps occupation dynamics, Eqs.(2.4) to (2.6):

$$
\begin{aligned}
\nabla \cdot(\varepsilon \nabla \phi) & =-q\left(p-n+N_{D}\left(1-f_{D}^{n}\right)-N_{A} f_{A}^{n}\right)-q \sum_{j} N_{t j}\left(\delta_{j}-f_{t j}^{n}\right) \\
\frac{\partial n}{\partial t}-N_{D} \frac{\partial f_{D}^{n}}{\partial t} & =\left(G_{n e t, n}-\sum_{j} R_{n}^{t j}-R_{a u}\right)+\frac{1}{q} \nabla \cdot \overrightarrow{J_{n}} \\
\frac{\partial p}{\partial t}+N_{A} \frac{\partial f_{A}^{n}}{\partial t} & =\left(G_{n e t, p}-\sum_{j} R_{p}^{t j}-R_{a u}\right)-\frac{1}{q} \nabla \cdot \overrightarrow{J_{p}} \\
N_{t j} \frac{\partial f_{t j}^{n}}{\partial t} & =R_{n}^{t j}-R_{p}^{t j} \quad \text { for each trap j, where: } \\
R_{n}^{t j} & =c_{n j} n N_{t j}\left(1-f_{t j}^{n}\right)-e_{n j} N_{t j} f_{t j}^{n} \\
R_{p}^{t j} & =c_{p j} p N_{t j} f_{t j}^{n}-e_{p j} N_{t j}\left(1-f_{t j}^{n}\right)
\end{aligned}
$$

where $f_{t j}^{n}$ means the electron occupation fraction of trap $j$ (in general $f_{j}^{n}=1-f_{j}^{p}$ for every trap $j$ with $f_{j}^{p}$ the complementary hole occupation fraction of the trap). The symbols $f_{D}^{n}, f_{A}^{n}$ are the occupancies for dopant donors and acceptors (if dopants are totally ionized, $f_{D}^{n}=0, f_{A}^{n}=1$ ). Even in the absence of traps coming from defects, Si has an indirect bandgap so a deep trap in the intrinsic level is considered to take into account phonon assisted recombination (the typical SRH recombination term, see [14]). $R_{a u}$ is the Auger recombination and $R_{n}^{t j}, R_{p}^{t j}$ are the electron and hole recombination rates for the trap $j . G_{n e t, n}, G_{n e t, p}$ are the net generation rates for electrons and holes, including optical, radiation, impact ionization and other available generation mechanisms. The summation term in Eq.(2.1) is $\rho_{\text {trap }}$, the trapped net charge, where $\delta_{j}=1$ if the trap $\mathrm{j}$ is of donor type, $\delta_{j}=0$ if the trap $\mathrm{j}$ is of acceptor type. In Eqs. $(2.5,2.6) c_{n j}, c_{p j}$ are the electron/hole capture terms and $e_{n j}, e_{p j}$ are the electron/hole emission terms for trap $j$.

The different transport models have different expressions to calculate $\vec{J}_{n}$ and $\vec{J}_{p}$. Sentaurus Device presents four models: Drift-Diffusion (DD), ThermoDynamic (TD), HydroDynamic (HD) and MonteCarlo (MC) [15]. DD is appropriate for simulations of low power devices with long active regions, as silicon particle detectors, in isothermal conditions. For semiconductors it is common to define Quasi-Fermi potentials, $\Phi_{n}, \Phi_{p}[16]$ to represent the carrier distribution functions which are slightly out of equilibrium so the DD currents [17], are:

$$
\vec{J}_{n}=-n q \mu_{n} \nabla \Phi_{n}=\mu_{n}\left(n \nabla E_{c}-\frac{3}{2} n K T \nabla \ln m_{n}\right)+D_{n}\left(\nabla n-n \nabla \ln \gamma_{n}\right)
$$

\footnotetext{
${ }^{5}$ particularized here to Silicon
} 


$$
\vec{J}_{p}=-p q \mu_{p} \nabla \Phi_{p}=\mu_{p}\left(n \nabla E_{v}-\frac{3}{2} n K T \nabla \ln m_{p}\right)-D_{p}\left(\nabla p-p \nabla \ln \gamma_{p}\right)
$$

where Sentaurus takes into account the contribution from drift, diffusion and also spatial variations of the effective mass and Fermi-Dirac statistics (the Fermi-Dirac degeneracy terms, $\gamma_{n}, \gamma_{p}$ are equal to 1 by using Maxwell-Boltzmann statistics, appropriate for non-degenerate semiconductors).

In order to get a glimpse of the modifications added to the semiconductor equations, let us consider a simple 2 trap model (acceptor and donor), like the classical EVL [18] defined with 8 parameters: trap energy levels $\left(E_{a}, E_{d}\right)$, concentrations $\left(N_{a}, N_{d}\right)$ and capture cross sections $\left(\sigma_{e}^{a}\right.$, $\sigma_{h}^{a}, \sigma_{e}^{d}, \sigma_{h}^{d}$ ). For our two trap model, we need to solve the trap occupation dynamics, $d f_{a}^{n} / d t$, $d f_{d}^{p} / d t$, where $f_{a}^{n}$ means electron trap occupation fraction of an acceptor trap, $f_{d}^{p}$ means hole trap occupation fraction of a donor trap. Now the Poisson equation has an explicit $\rho_{\text {trap }}$, Eq.(2.9). In steady state, the trap occupancies converge to $(2.10,2.11)$, with effective trapping times given by (2.12) and the net recombination equations for the two traps converge to a SRH type term, Eq.(2.13):

$$
\begin{aligned}
\rho_{\text {trap }}= & q\left[N_{d} f_{d}^{p}-N_{a} f_{a}^{n}\right] \\
f_{d}^{p}= & \frac{v_{h} \sigma_{h}^{d} p+v_{e} \sigma_{e}^{d} N_{c} e^{\left(E_{d}-E_{c}\right) / k T}}{v_{e} \sigma_{e}^{d}\left(n+N_{c} e^{\left(E_{d}-E_{c}\right) / k T}\right)+v_{h} \sigma_{h}^{d}\left(p+N_{v} e^{\left(E_{v}-E_{d}\right) / k T}\right)} \\
f_{a}^{p}= & \frac{v_{e} \sigma_{e}^{a} n+v_{h} \sigma_{h}^{a} N_{v} e^{\left(E_{v}-E_{a}\right) / k T}}{v_{e} \sigma_{e}^{a}\left(n+N_{c} e^{\left(E_{a}-E_{c}\right) / k T}\right)+v_{h} \sigma_{h}^{a}\left(p+N_{v} e^{\left(E_{v}-E_{a}\right) / k T}\right)} \\
\Gamma_{h}= & \frac{1}{\tau_{e f f, h}}=v_{h}\left[\sigma_{h}^{d} N_{d}\left(1-f_{d}^{p}\right)+\sigma_{h}^{a} N_{a} f_{a}^{n}\right] \quad ; \quad \Gamma_{e}=\frac{1}{\tau_{e f f, e}}=v_{e}\left[\sigma_{e}^{a} N_{a}\left(1-f_{a}^{n}\right)+\sigma_{e}^{d} N_{d} f_{d}^{p}\right] \\
R_{n e t}= & \frac{v_{h} v_{e} \sigma_{h}^{d} \sigma_{e}^{d} N_{d}\left(n p-n_{i}^{2}\right)}{v_{e} \sigma_{e}^{d}\left(n+N_{c} e^{\left(E_{d}-E_{c}\right) / k T}\right)+v_{h} \sigma_{h}^{d}\left(p+N_{v} e^{\left(E_{v}-E_{d}\right) / k T}\right)} \\
& +\frac{v_{h} v_{e} \sigma_{h}^{a} \sigma_{e}^{a} N_{a}\left(n p-n_{i}^{2}\right)}{v_{e} \sigma_{e}^{a}\left(n+N_{c} e^{\left(E_{a}-E_{c}\right) / k T}\right)+v_{h} \sigma_{h}^{a}\left(p+N_{v} e^{\left(E_{v}-E_{a}\right) / k T}\right)}
\end{aligned}
$$

From the previous exposition of the mathematics involved in a TCAD simulation it is clear that the full set of traps coming from physical studies, for example given in [19], is non-practical in terms of computing resources. It is compulsory to choose an effective set of traps for modeling the measured and identified point and cluster defects. The mobility has also to take into account the traps (in Sentaurus, for example, using the Philips mobility model), specially when the detector is not fully depleted (i.e. when the carrier velocities are not saturated). Last but not least, the SRH trap statistics is not optimal in case of clusters so a parameter tweaking is also needed, in particular for deep traps that are responsible for the leakage current generation.

\section{High fluence models}

Recently the community presented several radiation damage models adjusted for the expected HL-LHC fluence. Fluence, $\Phi$ is linearly related to trap density, $N\left(\mathrm{~cm}^{-3}\right)=\eta \Phi$, where $\eta$ is the introduction rate. The three models discussed here use the Van Overstraeten-De Man avalanche model for impact ionization effects due to the high field strength in highly irradiated detectors (other 


\begin{tabular}{|c|c|c|c|c|c|}
\hline Defect Number & Type & Energy level $(\mathrm{eV})$ & $\sigma_{e}\left[\mathrm{~cm}^{-2}\right]$ & $\sigma_{h}\left[\mathrm{~cm}^{-2}\right]$ & $\eta\left[\mathrm{cm}^{-1}\right]$ \\
\hline 1 & Donor & $E_{v}+0.48$ & $2 \times 10^{-14}$ & $1 \times 10^{-14}$ & 4 \\
\hline 2 & Acceptor & $E_{c}-0.525$ & $5 \times 10^{-15}$ & $1 \times 10^{-14}$ & 0.75 \\
\hline 3 & Acceptor & $E_{v}+0.90$ & $1 \times 10^{-16}$ & $1 \times 10^{-16}$ & 36 \\
\hline
\end{tabular}

Table 1: LHCb high fluence defect model [20].

\begin{tabular}{|c|c|c|c|c|c|c|c|c|}
\hline Type & Energy level $(\mathrm{eV})$ & \multicolumn{3}{|c|}{$\sigma_{e}\left(\mathrm{~cm}^{-2}\right)$} & \multicolumn{3}{c|}{$\sigma_{h}\left(\mathrm{~cm}^{-2}\right)$} \\
\hline & & $\mathrm{r} 1$ & $\mathrm{r} 2$ & $\mathrm{r} 3$ & $\mathrm{r} 1$ & $\mathrm{r} 2$ & $\mathrm{r} 3$ \\
\hline Acceptor & $E_{c}-0.42$ & $1 \times 10^{-15}$ & $1 \times 10^{-15}$ & $1 \times 10^{-15}$ & $1 \times 10^{-14}$ & $1 \times 10^{-14}$ & $1 \times 10^{-14}$ & 1.613 \\
\hline Acceptor & $E_{c}-0.46$ & $7 \times 10^{-15}$ & $3 \times 10^{-15}$ & $1.5 \times 10^{-15}$ & $7 \times 10^{-14}$ & $3 \times 10^{-14}$ & $1.5 \times 10^{-14}$ & 0.9 \\
\hline Donor & $E_{v}+0.36$ & $3.23 \times 10^{-13}$ & $3.23 \times 10^{-13}$ & $3.23 \times 10^{-13}$ & $3.23 \times 10^{-14}$ & $3.23 \times 10^{-14}$ & $3.23 \times 10^{-14}$ & 0.9 \\
\hline
\end{tabular}

Table 2: "New Perugia" bulk damage, for three fluence ranges, r1:up to $7 \times 10^{15} \mathrm{n}_{\mathrm{eq}} / \mathrm{cm}^{2}, \mathrm{r} 2: 7 \times 10^{15}$ $2.2 \times 10^{16} \mathrm{n}_{\mathrm{eq}} / \mathrm{cm}^{2}$ and $\mathrm{r} 3: 1.6 \times 10^{16}-2.2 \times 10^{16} \mathrm{n}_{\mathrm{eq}} / \mathrm{cm}^{2}$. For a given fluence range there is a capture cross section value associated for electrons and holes [21].

\begin{tabular}{|c|c|c|}
\hline Interface Defect & Level(eV) & Concentration \\
\hline Acceptor & $E_{c}-0.4$ & $N_{i t}=0.4 \times 0.85 \times N_{o x}$ \\
\hline Acceptor & $E_{c}-0.6$ & $N_{i t}=0.6 \times 0.85 \times N_{o x}$ \\
\hline Donor & $E_{v}+0.7$ & $N_{i t}=0.85 \times N_{o x}$ \\
\hline
\end{tabular}

Table 3: "New Perugia" Interface Damage (oxide charge density $N_{o x}$, interface trap density $N_{i t}$ ) [22].

impact ionization models give 3-4\% variation in CCE). The simplest one is explained in [20], from the LHCb collaboration, and applied to VELO pixel detectors, valid up to $8 \times 10^{15} \mathrm{n}_{\mathrm{eq}} / \mathrm{cm}^{2}$. They add a third acceptor level ( 3 in Table 1) to the EVL model. Cross sections are adjusted to experimental results, with measurements from $200 \mu m$ thick n-on-p sensors bump bonded to a TimePix 3 readout chip. The model is able to capture the transition from a linear electric field/saturating I-V curve to a double junction electric field/non-saturating I-V curve as a consequence of avalanche generation in the high field regions of double junctions. For a center pixel hit the estimated CCE has less than $10 \%$ of error compared with the measurements.

The "New Perugia" model, presented in 2015, [21, 22] is appropriate for higher fluences (up to $2.2 \times 10^{16} \mathrm{n}_{\mathrm{eq}} / \mathrm{cm}^{2}$ ), with one set of parameters for fluences up to $7 \times 10^{15} \mathrm{n}_{\mathrm{eq}} / \mathrm{cm}^{2}$ and for the range $7 \times 10^{15}-2.2 \times 10^{16} \mathrm{n}_{\mathrm{eq}} / \mathrm{cm}^{2}$. It includes a modeling of bulk and also surface damage in the $\mathrm{Si}-\mathrm{SiO}_{2}$ interface (in case of microelectronics or AC coupled detectors). The bulk model, Table (2), derives from the "Old Perugia" model [23] and a literature survey made by the Perugia group. The surface model, Table (3), was obtained from experimental measurements on gated diodes and MOS capacitors, p-type substrate after $\gamma$ irradiation (50-100 Mrad).

The latest damage model is the Hamburg PentaTrap Model (HPTM) (2018 [24]), see Table 4. It intends to describe at the same time I-V, C-V and CCE measurements on pad diodes irradiated with $24 \mathrm{GeV} / \mathrm{c}$ protons with fluences $>10^{15} \mathrm{n}_{\mathrm{eq}} / \mathrm{cm}^{2}$. It is based on 5 traps, both cross sections for $\mathrm{E} 30 \mathrm{~K}$ and the electron cross section for $C_{i} O_{i}$ fixed and 12 free parameters adjusted to simulation by optimization with the non-linear simplex method. The Sentaurus TCAD optimizer minimize the relative deviation between the simulations and measurements over a large voltage 


\begin{tabular}{|c|c|c|c|c|c|}
\hline Defect & Type & Energy level $(\mathrm{eV})$ & $\eta\left[\mathrm{cm}^{-1}\right]$ & $\sigma_{e}\left[\mathrm{~cm}^{-2}\right]$ & $\sigma_{h}\left[\mathrm{~cm}^{-2}\right]$ \\
\hline$E 30 K$ & Donor & $E_{c}-0.1$ & 0.0497 & $2.300 \times 10^{-14}$ & $2.920 \times 10^{-16}$ \\
\hline$V_{3}$ & Acceptor & $E_{c}-0.458$ & 0.6447 & $2.551 \times 10^{-14}$ & $1.511 \times 10^{-13}$ \\
\hline$I_{p}$ & Acceptor & $E_{c}-0.545$ & 0.4335 & $4.478 \times 10^{-15}$ & $6.790 \times 10^{-15}$ \\
\hline$H 220$ & Donor & $E_{v}+0.48$ & 0.5978 & $4.166 \times 10^{-15}$ & $1.965 \times 10^{-16}$ \\
\hline$C_{i} O_{i}$ & Donor & $E_{v}+0.36$ & 0.3780 & $3.230 \times 10^{-17}$ & $2.036 \times 10^{-14}$ \\
\hline
\end{tabular}

Table 4: Hamburg Pentatrap Model [24].

range, specifically it minimizes $F$ in Eq.(3.1):

$$
F=\sum_{i, j} w_{i}^{j} \int_{V_{\min }}^{V_{\max }}\left(1-\frac{Q_{i, \text { sim }}^{j}}{Q_{i, \text { meas }}^{j}}\right)^{2} d V
$$

where $i$ runs over different fluences, $j$ runs over the different measurements with $Q_{i, \text { sim }}^{j}$ and $Q_{i, \text { meas }}^{j}$ are the simulated and measured quantities (currents, capacitances and CCE's). $V_{\min }, V_{\max }$ are the minimum and maximum voltages and $w_{i}^{j}$ are weighting factors to weight the different kind of measurements. The simulations for optimization were made at $-20^{\circ} \mathrm{C}$ with Slotboom bandgap narrowing, TAT Hurkx with tunnel mass of $0.25 m_{e}$ (default value $0.5 m_{e}$ ) for defect $I_{p}$, relative permittivity of Silicon 11.9 (default value 11.7). The calibration measurements were made on $2 \times 2$ and $5 \times 5 \mathrm{~mm}^{2} 200 \mu \mathrm{m}$ thick float zone p-type pad diodes (with p-stop and p-spray). The electrical characterization was made after $80 \mathrm{~min}$ of annealing at $60^{\circ} \mathrm{C}$. Measurements were performed at $-20^{\circ} \mathrm{C}$, consisting in I-V up to $1000 \mathrm{~V}$ (reverse) and up to a current limit of $0.5 \mathrm{~mA}$ (forward), $\mathrm{C} / \mathrm{G}-\mathrm{V}$ measurements with $100 \mathrm{~Hz}-2 \mathrm{MHz}$ of excitation frequency and laser TCT measurements at $670 \mathrm{~nm}$ (red) and $1064 \mathrm{~nm}$ (IR) wavelengths.

The I-V/C-V and the CCE-V simulations agree with the measurement results within $20 \%$ for all fluences $\left(0.3\right.$ to $\left.13 \times 10^{15} \mathrm{n}_{\mathrm{eq}} / \mathrm{cm}^{2}\right)$ in the voltage range (-1000 to $\left.0 \mathrm{~V}\right)$. It also reproduces the double peak structure (E-field vs position) for fluences $\geq 3 \times 10^{15} \mathrm{n}_{\text {eq }} / \mathrm{cm}^{2}$, with a peak field of $\simeq 2 \times 10^{5} \mathrm{~V} / \mathrm{cm}$ at the highest fluence, responsible for impact ionization.

The previous defect models were not able to simulate the acceptor removal effect, specially relevant in LGAD-type devices [25]. At the present state of knowledge, we use Eq.(1.1) to redefine the p-gain layer doping profile, $N_{A, p_{\text {gain }}}$ in the device model previous to the simulation. A full simulation of an irradiated LGAD-type sensor also has to include a defect model to take into account the traps introduced due to radiation.

\section{Simulation Examples}

As an example of the combination of the "New Perugia" model with the acceptor removal effect, we present a simulation effort for LGAD devices [26] made with TCAD Sentaurus from Synopsys, see Table 5 . The objective for that work was to show that radiation damage in LGAD can reduce the charge gain when approaching to high fluences due to the acceptor removal effect (with minor influence due to the appearance of the double junction effect). For that work we made a 2D simulation of a $300 \mu \mathrm{m}$ thick LGAD, $5 \mathrm{~mm}$ length, biased to $400 \mathrm{~V}$, at $253 \mathrm{~K}$ with an acceptor removal constant $c=4 \times 10^{-16} \mathrm{~cm}^{2}$. The detector model excitation was a red laser pulse, 


\begin{tabular}{|c|c|c|c|}
\hline Fluence $\mathrm{n} / \mathrm{cm}^{2}$ & Charge LGAD(C) & Charge PIN(C) & Gain $Q_{\text {lgad }} / Q_{\text {pin }}$ \\
\hline No Irrad & $9.86 \mathrm{e}-15$ & $2.01 \mathrm{e}-15$ & 4.91 \\
\hline $1 \times 10^{13}$ & $9.46 \mathrm{e}-15$ & $2.00 \mathrm{e}-15$ & 4.72 \\
\hline $1 \times 10^{14}$ & $6.77 \mathrm{e}-15$ & $1.95 \mathrm{e}-15$ & 3.46 \\
\hline $1 \times 10^{15}$ & $1.74 \mathrm{e}-15$ & $1.22 \mathrm{e}-15$ & 1.42 \\
\hline $2 \times 10^{15}$ & $1.28 \mathrm{e}-15$ & $1.19 \mathrm{e}-15$ & 1.08 \\
\hline
\end{tabular}

Table 5: LGAD simulation example [26].

illuminating through the $\mathrm{p}^{++}$layer, $\lambda=670 \mathrm{~nm}, 10 \mu \mathrm{m}$ of spot radius, $50 \mathrm{~W} / \mathrm{cm}^{2}$ and $200 \mathrm{ps}$ of duration. We compared the simulations of the LGAD (Van Overstraeten-De Man avalanche model) with its associated PIN (same device but without the gain p-layer), defining the gain as the quotient of the cumulated charges, Gain $=Q_{\text {lgad }} / Q_{\text {pin }}$.

The LGAD device evolved to the iLGAD [27]. The iLGAD is a strip detector with intrinsic gain generated in a gain layer on the non-segmented side of the sensor. Conventional segmentation of a LGAD, at the $\mathrm{n}+$ ohmic side, means also non-uniformities in the multiplication. The iLGAD has the segmentation at the $\mathrm{p}+$ ohmic side so the p-multiplication layer is continuous below the $\mathrm{n}+$ ohmic side. The CCE simulation [28] showed the iLGAD at room temperature (300K, "New Perugia" defect model) and at the expected operation temperature (HPTM defect model, specifically appropriated at $253 \mathrm{~K}$ ), with $300 \mathrm{~V}$ bias, under same laser excitation (red laser hit at the right center strip segment) and higher damage fluences (acceptor removal constant $c=4 \times 10^{-16} \mathrm{~cm}^{2}$ ). The results are shown in Table 6 where we see an improvement of the CCE at 253K. The agreement with measurements is around $20 \%$.

The last example, presented at [29] and also made with TCAD Sentaurus, simulates a monolithic detector called OVERMOS. It is a CMOS Monolithic Active Pixel Sensor (MAPS) fabricated with the Tower Jazz $180 \mathrm{~nm}$ technology kit. The novelty of this simulation is it also has to take into account TID effects because there are dielectrics $\left(\mathrm{SiO}_{2}\right)$ over the active regions. In the simulation model the impact ionization was considered with the UniBo model to take into account possible avalanche effects due to a high concentration of defects. The model for defects is twofold: HPTM for the bulk region (with a factor of 1.66 multiplying all the HPTM introduction rates to account for neutron irradiation) and the "New Perugia" for the interface traps [22]. The TID model includes fixed oxide charge, $N_{o x}$ and interface traps, $N_{i t}$ with $N_{o x}=1.2 \times 10^{11} \mathrm{~cm}^{-3}$ and $N_{i t}=0.85 \times N_{o x}$. The interface traps have a gaussian distribution with $\sigma=0.7 \mathrm{eV}$ and a cross-section of $1 \times 10^{-15} \mathrm{~cm}^{-2}$.

The breakdown voltage, defined as $(\Delta I / \Delta V)_{\max }$, shows an agreement up to $\sim 4 \mathrm{~V}$. The I-V simulations show $\mathrm{a} \sim 30 \%$ agreement with the measurements, for fluences in the range $10^{13}-10^{15}$ $\mathrm{n}_{\mathrm{eq}} / \mathrm{cm}^{2}$. For CCE simulations using laser injection (center pixel hit, $\lambda=1064 \mathrm{~nm}$, pulse energy 25 pJ, pulse width $7.8 \mathrm{~ns}$, laser window $5 \times 5 \mu \mathrm{m}^{2}$ ) the agreement is acceptable between the measured and simulated collected charge, $Q_{\text {coll }}$, as shown in Table 7.

\section{Conclusions}

Simulation of irradiated sensors under HL-LHC expected high fluences is an ongoing work in the RD50 collaboration. The TCAD software is well understood, specially in the case of TCAD 


\begin{tabular}{|c|c|c|c|c|}
\hline Fluence $\mathrm{n} / \mathrm{cm}^{2}$ & Charge(fC)(300K) & \% reduction & Charge $(\mathrm{fC})(253 \mathrm{~K})$ & \% reduction \\
\hline No Irrad & $8.24 \times 10^{-1}$ & - & 1.08 & - \\
\hline $1 \times 10^{14}$ & $5.26 \times 10^{-1}$ & $63.8 \%$ & $7.41 \times 10^{-1}$ & $68.6 \%$ \\
\hline $1 \times 10^{15}$ & $2.79 \times 10^{-1}$ & $33.8 \%$ & $5.67 \times 10^{-1}$ & $52.5 \%$ \\
\hline $7.5 \times 10^{15}$ & $6.69 \times 10^{-2}$ & $8.1 \%$ & $1.5 \times 10^{-1}$ & $13.8 \%$ \\
\hline
\end{tabular}

Table 6: iLGAD simulation example [28]"New Perugia" defect model for 300K, HPTM defect model for $253 \mathrm{~K}$, acceptor removal constant $c=4 \times 10^{-16} \mathrm{~cm}^{2}$, red laser: $\lambda=670 \mathrm{~nm}, 10 \mu \mathrm{m}$ of spot radius, $50 \mathrm{~W} / \mathrm{cm}^{2}$, 200 ps of pulse width..

\begin{tabular}{|c|c|c|c|}
\hline Fluence $\left(n_{e q} / \mathrm{cm}^{2}\right)$ & $Q_{\text {coll }}(\mathrm{fC})$ Test & $Q_{\text {coll }}(f C)$ Simulation & $\Delta \%$ \\
\hline No Irrad & 153 & 166 & -8.4 \\
\hline $1 \times 10^{13}$ & 110 & 143 & -30 \\
\hline $5 \times 10^{13}$ & 106 & 82 & 22 \\
\hline $1 \times 10^{14}$ & 65 & 53 & 18 \\
\hline $5 \times 10^{14}$ & 27 & 23 & -14 \\
\hline $1 \times 10^{15}$ & 10 & 17 & 70 \\
\hline
\end{tabular}

Table 7: CCE OVERMOS pixel simulation, $1064 \mathrm{~nm}$ laser hit [29].

Sentaurus from Synopsys, and incremental improvements has been added by means of tailor made functions. The available defect models give a reasonable precision in terms of general behavior but with errors around $20 \%$ when compared with measurements for bulk devices. The simulations for non-irradiated MAPS devices show the same 20\% agreement but there are bigger discrepancies for the irradiated ones. Besides that, every device needs a specific defect modeling, for example acceptor removal effect for the LGAD family or surface defects for MAPS. TCAD simulation is an excellent tool to understand the device behavior under radiation damage but the predictive power needs to be improved. The RD50 collaboration is actively working in that direction.

\section{References}

[1] The Phase-2 upgrade of the CMS Tracker, Tech. Rep. CERN-LHCC-2017-009, CMS-TDR-014.

[2] I.Pintilie et al., Radiation-induced point and cluster defects with strong impact on damage properties of silicon detectors, Nuclear Instruments and Methods in Physics Research A, 611, (2009), pp.52-68.

[3] M.Moll, Displacement damage in silicon detectors for high energy physics, IEEE Transactions on Nuclear Science, 65(8), 2018, pp.1561-1582.

[4] K.Gill, G.Hall, B.MacEvoy, Bulk damage effects in irradiated silicon detectors due to clustered divacancies, Journal of Applied Physics, 82(1), 1997, pp.126-136.

[5] A.Schenk, U.Krumbein, Coupled defect-level recombination: Theory and application to anomalous diode characteristics, Journal of Applied Physics, 78(5), 1995, pp.3185-3192.

[6] A.Scheinemann, A.Schenk, TCAD-based DLTS simulation for analysis of extended defects, Physica Status Solidi A 211(1), 2014, pp.136-242.

[7] S.J.Watts et al., A new model for generation-recombination in silicon depletion regions after neutron irradiation, IEEE Transactions on Nuclear Science, 43(6), 1996, pp.2587-2594. 
[8] M.Ferrero et al., Radiation resistant LGAD design, Nuclear Instrumens and Methods in Physics Research A, 919,(2019), pp.16-26.

[9] Y.Gurimskaya et al., Radiation damage in p-type EPI silicon pad diodes irradiated with protons and neutrons, Nuclear Instruments and Methods in Physics Research A (2019), in press.

[10] M.Moll, Acceptor removal-Displacement damage effects involving the shallow acceptor doping of p-type silicon devices, Proceedings of Science (Vertex2019), 27, 2020.

[11] P.Fernández-Martínez et al.,Low Gain Avalanche Detectors for high energy physics, 2015 10th Spanish Conference on Electron Devices (CDE), 20 April 2015, Aranjuez, Spain, pp. 1-4, http://www.cde-conf.org/cde15/cde2015/, doi: 10.1109/CDE.2015.7087475.

[12] CMOS Monolithic Active Pixel Detectors (MAPS) for future vertex detectors, R.Turchetta, Journal of Instrumentation, 1, P08004, 2006.

[13] S.Li and Y.Fu, 3D TCAD Simulation for Semiconductor Processes, Devices and Optoelectronics, Springer 2012.

[14] S.M.Sze, Semiconductor Devices, Physics and Technology, John Wiley and Sons, 1985.

[15] Sentaurus Device User Guide, Version O-2018.06, June 2018.

[16] R.F.Pierret, Advanced Semiconductor Fundamentals, 2nd.ed., Modular Series on Solid State Devices, 2002.

[17] K.M.Chang, A consistent model for carrier transport in heavily doped semiconductor devices, Semiconductor Science and Technology, 3(8), 1988, pp.766-772.

[18] V.Eremin, Z.Li, S.Roe, G.Ruggiero, E.Verbitskaya, Double peak electric field distortion in heavily irradiated silicon strip detectors, Nuclear Instruments in Physics Research A, 535, 2004, pp.622-631.

[19] A.Junkes, Status of Defect investigations, Proceedings of Science (Vertex 2011), 035, 2011.

[20] ̊̊.Folkestad et al., Development of a silicon bulk radiation damage model for Sentaurus TCAD, Nuclear Instruments in Physics Research A, 874, 2017, pp.94-102.

[21] F.Moscatelli et al., Combined bulk and surface effects at very high fluences in silicon detectors: measurements and TCAD simulations, IEEE Transactions on Nuclear Science, 63(5), 2016, pp.2716-2723.

[22] F.Moscatelli et al., Effects of interface donor trap states on isolation properties of detectors operating at the High-Luminosity LHC, IEEE Transactions on Nuclear Science, 64(8), 2017.

[23] M.Petasecca et al., Numerical simulation of radiation damage effects in p-type and n-type FZ silicon detectors, IEEE Transactions on Nuclear Science, 53(5), 2006, pp.2971-2976.

[24] J.Schwandt et al., A new model for the TCAD simulation of the silicon damage by high fluence proton irradiation, 2018 IEEE Nuclear Science Symposium and Medical Imaging Conference Proceedings (NSS/MIC), 10-17 Nov.2018, Sidney, Australia, pp. 1-3, http: //www.nssmic.org/2018, doi: 10.1109/NSSMIC.2018.8824412

[25] G. Kramberger et al., Radiation effects in low gain avalanche detectors after hadron irradiations, Journal of Instrumentation 10, P07006, 2015.

[26] F.R.Palomo, S.Hidalgo, LGAD simulations with Ga doping: an exploration, 30th RD50 Workshop, https://indico.cern.ch/event/637212/, 5-7th June 2017, Krakow, Poland. 
[27] G.Pellegrini et al., Recent technological developments on LGAD and iLGAD detectors for tracking and timing applications, Nuclear Instruments and Methods in Physics Research A, 831 (2016), pp.24-28.

[28] F.R.Palomo, S.Hidalgo, I.Vila, ILGAD TCAD Simulations: first approximations, 32nd RD50 Workshop, https: / / indico. cern. ch/event/719814/, 4-6th June 2018, Hamburg, Germany.

[29] E.G.Villani, TCAD Processes and device simulations of OVERMOS, a CMOS 180nm MAPS detector, 34th RD50 Workshop, https: / / indico.cern. ch/event/812761/, Lancaster University, UK, 12th-14th June 2019. 